\title{
POSITIVE SOLUTIONS FOR NONLINEAR ELASTIC BEAM MODELS
}

\author{
BENDONG LOU
}

(Received 14 December 1999)

\begin{abstract}
We give a negative answer to a conjecture of Korman on nonlinear elastic beam models. Moreover, by modifying the main conditions in the conjecture (generalizing the original ones at some points), we get positive results, that is, we obtain the existence of positive solutions for the models.
\end{abstract}

2000 Mathematics Subject Classification. 34B15.

1. Introduction. In 1988, Korman [3] studied the nonlinear elastic beam models

$$
\begin{gathered}
u^{\prime \prime \prime \prime}=f(x, u), \quad 0<x<1, \\
u(0)=\alpha, \quad u^{\prime}(0)=\beta, \quad u(1)=\gamma, \quad-u^{\prime}(1)=\delta,
\end{gathered}
$$

where $\alpha, \beta, \gamma, \delta \geq 0, f:[0,1] \times \mathbb{R}_{+} \rightarrow \mathbb{R}_{+}$is continuous. Equation $u^{\prime \prime \prime \prime}=f(x, u)$ with appropriate boundary conditions describes various physically important boundary value problems (see [3]). Korman [3] studied the existence of positive solutions of (1.1) by using monotone iterations. His results covered in particular the sublinear nonlinearities. For the superlinear case, he discussed one special case and stated the following conjecture.

CONJECTURE 1.1. Let $f(x, u)$ be increasing in $u$ for $0 \leq u<\infty, 0 \leq x \leq 1, f(x, u)>$ 0 for $u>0$, and (uniformly in $x$ )

$$
\lim _{u \rightarrow 0^{+}} \frac{f(x, u)}{u}=0, \quad \lim _{u \rightarrow+\infty} \frac{f(x, u)}{u}=+\infty .
$$

Then problem (1.1) has a positive solution.

In this paper, we give a negative answer to this conjecture by constructing a counterexample. Moreover, we give a general sufficient condition to guarantee the existence of positive solutions of (1.1), precisely to say, we modify the equalities in (1.2) a little (see (3.4) in Section 3), and then using topological degree theory and cone theory to prove that (1.1) has at least one positive solution under this modified condition, and that the other conditions in the conjecture are unnecessary.

2. Some lemmas. In what follows, denote by $\|\cdot\|$ the supremum norm of the Banach space $C[0,1]$. Let $P=\{u \in C[0,1] \mid u(x) \geq 0$ for $x \in[0,1]\}$, then $P$ is a cone of $C[0,1]$ and it induces a partial ordering in $C[0,1]$, that is, $u \leq v$ if and only if $u(x) \leq v(x)$ for $x \in[0,1]$ (cf. [1, 2]). Denote by $U_{l}=\{u \in C[0,1] \mid\|u\|<l\}(l>0)$, the open ball in $C[0,1]$. 
LEMMA 2.1. For $u \in C^{4}[0,1], u$ is a solution of (1.1) if and only if $u \in C[0,1]$ is a solution of the integral equation

$$
u(x)=A u(x) \equiv \xi(x)+B u(x)
$$

where

$$
\begin{gathered}
\xi(x)=\alpha(1-x)^{2}(2 x+1)+\beta x(1-x)^{2}+\gamma x^{2}(3-2 x)+\delta x^{2}(1-x), \\
B u(x)=\int_{0}^{1} G(x, y) f(y, u(y)) d y,
\end{gathered}
$$

where $G(x, y)$ is the Green's function for $u^{\prime \prime \prime \prime}$ with the boundary conditions $u(0)=$ $u^{\prime}(0)=u(1)=u^{\prime}(1)=0$, that is,

$$
G(x, y)= \begin{cases}\frac{x^{2}}{6}(1-y)^{2}(3 y-2 x y-x), & 0 \leq x \leq y \leq 1, \\ \frac{y^{2}}{6}(1-x)^{2}(3 x-2 x y-y), & 0 \leq y \leq x \leq 1 .\end{cases}
$$

Proof. The conclusion can be verified in a standard way.

REMARK 2.2. It is clear that $G(x, y)$ is continuous on $[0,1] \times[0,1], G(x, y)=$ $G(y, x)$ for $0 \leq x, y \leq 1$, and $G(x, y)>0$ for $0<x, y<1$.

LEMMA 2.3. Let $G(x, y)$ be defined by (2.4), then

$$
G(x, y) \geq \frac{2}{3} x^{2}(1-x)^{2} G(z, y), \quad \forall x, y, z \in[0,1] .
$$

\section{ProOF}

CASE $1(0 \leq x \leq y \leq 1)$. (1) If $z \leq y$, then

$$
\begin{aligned}
G(x, y) & =\frac{x^{2}}{6}(1-y)^{2}(3 y-2 x y-x) \geq \frac{x^{2}}{6}(1-y)^{2} \cdot 2 y(1-x) \\
& \geq 2 x^{2}(1-x) \frac{(1-y)^{2}}{6} y \cdot \frac{z(3 y-2 y z-z)}{3} \\
& \geq \frac{2 x^{2}(1-x)}{3} G(z, y) .
\end{aligned}
$$

(2) If $y \leq z$, then

$$
\begin{aligned}
G(x, y) & =\frac{x^{2}}{6}(1-y)^{2}(3 y-2 x y-x) \geq \frac{2 x^{2}(1-x)}{6}(1-y)^{2} y \\
& \geq \frac{2 x^{2}(1-x)}{6}(1-z)^{2} y \cdot \frac{y(3 z-2 y z-y)}{3} \\
& =\frac{2 x^{2}(1-x)}{3} G(z, y) .
\end{aligned}
$$

CASE $2(0 \leq y \leq x \leq 1)$. It is easy to verify in a similar way as in Case 1

$$
G(x, y) \geq \frac{2 x(1-x)^{2}}{3} G(z, y), \quad \forall x, y, z \in[0,1], y \leq x .
$$


Hence,

$$
G(x, y) \geq \frac{2 x^{2}(1-x)^{2}}{3} G(z, y), \quad \forall x, y, z \in[0,1] .
$$

This completes the proof.

LEMMA 2.4. There exists $0<\sigma_{1} \leq 1$ such that

$$
\xi(x) \geq \frac{2 \sigma_{1}}{3} x^{2}(1-x)^{2} \xi(y), \quad \forall x, y \in[0,1] .
$$

Proof. By (2.2), it is easy to see that

$$
0 \leq \xi(x) \leq \alpha+\frac{4}{27} \beta+\gamma+\frac{4}{27} \delta \equiv \omega, \quad \forall x \in[0,1] .
$$

If $\omega=0$, then $\xi(x) \equiv 0$ and (2.10) is satisfied for any $0<\sigma_{1} \leq 1$.

We now suppose that $\omega>0$. If $\alpha>0$, then choose $0<\sigma_{2} \leq \min \{1,3 \alpha / 2 \omega\}$, we have

$$
\begin{aligned}
\xi(x) & \geq \alpha(1-x)^{2}(2 x+1) \geq \alpha(1-x)^{2} \geq \frac{2}{3} \omega \sigma_{2}(1-x)^{2} \\
& \geq \sigma_{2} \cdot \frac{2}{3} x^{2}(1-x)^{2} \omega \geq \frac{2 \sigma_{2}}{3} x^{2}(1-x)^{2} \xi(y), \quad \forall x, y \in[0,1] .
\end{aligned}
$$

If $\alpha=0, \beta>0$ (or $\gamma>0$, or $\delta>0$ ), the proof is similar. This completes the proof.

REMARK 2.5. Set

$$
v^{*}(x)=\frac{2 \sigma_{1}}{3} x^{2}(1-x)^{2} \equiv \sigma x^{2}(1-x)^{2},
$$

where $\sigma_{1}$ is given by (2.10). Then (2.5) remains valid if we replace $(2 / 3) x^{2}(1-x)^{2}$ by $v^{*}(x)$.

LEMMA 2.6. For any $u \in P$, there exists $\tau>0$ such that

$$
v^{*}(x) \geq \tau B u(x), \quad \forall x \in[0,1] .
$$

In particular, let $f \equiv 1$ in (2.3), then for some $\tau_{0}>0$,

$$
v^{*}(x) \geq \tau_{0} \int_{0}^{1} G(x, y) d y, \quad \forall x \in[0,1] .
$$

Proof. To prove (2.14) it suffices to prove that there exists $\tau>0$ such that

$$
p(x) \equiv x^{2}(1-x)^{2} \geq \tau \int_{0}^{1} G(x, y) f(y, u(y)) d y=\tau B u(x), \quad \forall x \in[0,1] .
$$

Since

$$
G(0, y)=0, \quad \frac{\partial G(0, y)}{\partial x}=0, \quad \frac{\partial^{2} G(0, y)}{\partial x^{2}}=y(1-y)^{2}, \quad \forall y \in[0,1],
$$

we have

$$
B u(0)=(B u)^{\prime}(0)=0, \quad(B u)^{\prime \prime}(0)=\int_{0}^{1} y(1-y)^{2} f(y, u(y)) d y \equiv \tau_{1} \geq 0 .
$$


On the other hand, $p(0)=p^{\prime}(0)=0, p^{\prime \prime}(0)=2>0$. Hence, there exists $\tau_{2}>0$ such that $p^{\prime \prime}(0)>\tau_{2}(B u)^{\prime \prime}(0)$. Thus, for some small $\delta_{1}>0$,

$$
p(x) \geq \tau_{2} B u(x), \quad \forall 0 \leq x \leq \delta_{1} .
$$

Similarly, there exist $\tau_{3}>0$ and $\delta_{2}>0$ sufficiently small such that

$$
p(x) \geq \tau_{3} B u(x), \quad \forall 1-\delta_{2} \leq x \leq 1 .
$$

Therefore, there exists $\tau>0$ such that (2.16) holds. So, Lemma 2.6 is proved.

Consider the linear eigenvalue problem

$$
u^{\prime \prime \prime \prime}=\lambda u, \quad 0<x<1, \quad u(0)=u^{\prime}(0)=u(1)=u^{\prime}(1)=0 .
$$

It is easy to see that (2.21) is equivalent to the following integral equation:

$$
u(x)=\lambda \int_{0}^{1} G(x, y) u(y) d y \equiv \lambda L u(x),
$$

where $G(x, y)$ is given by (2.4), and it is easy to verify by $G(x, y)>0(x, y \in(0,1))$ that the spectral radius of $L, r(L)$ is positive, therefore Krein-Rutman's theorem (see [4]) shows that there exists $\phi(x) \in P, \phi(x) \not \equiv 0$ and $\|\phi\|=1$ such that

$$
\phi(x)=\lambda_{1} \int_{0}^{1} G(x, y) \phi(y) d y,
$$

where $\lambda_{1}=r(L)^{-1}>0$ is the first eigenvalue of (2.21).

LEMmA 2.7. There exists $c \in(1 / 3,2 / 3)$ such that $\phi$ is strictly increasing on $[0, c]$ and strictly decreasing on $[c, 1]$. Therefore

$$
\phi(x) \geq \min \left\{\phi\left(\frac{1}{3}\right), \phi\left(\frac{2}{3}\right)\right\} \equiv \phi_{0}>0, \quad \forall \frac{1}{3}<x<\frac{2}{3} .
$$

Proof. By (2.23), we have

$$
\phi(0)=\phi(1)=0 ; \quad \phi(x)>0, \quad \forall 0<x<1 .
$$

By differentiating (2.23) we get

$$
\begin{aligned}
\phi^{\prime}(x)= & \lambda_{1} \int_{0}^{x} \frac{y^{2}}{2}(x-1)(3 x-2 x y-1) \phi(y) d y \\
& +\lambda_{1} \int_{x}^{1} \frac{x}{2}(y-1)^{2}(2 y-2 x y-x) \phi(y) d y \equiv p(x)+q(x) .
\end{aligned}
$$

Clearly, $p(0)=p(1)=0, q(0)=q(1)=0$. An elementary computation shows that

$$
\begin{aligned}
& p(x)>0, \quad \forall 0<x \leq \frac{1}{3} ; \quad p(x)<0, \quad \forall \frac{1}{2}<x<1 ; \\
& q(x)>0, \quad \forall 0<x<\frac{1}{2} ; \quad q(x)<0, \quad \forall \frac{2}{3}<x<1 .
\end{aligned}
$$


Differentiating (2.23) twice, we get

$$
\begin{aligned}
\phi^{\prime \prime}(x)= & \lambda_{1} \int_{0}^{x} y^{2}(3 x-2 x y+y-2) \phi(y) d y \\
& +\lambda_{1} \int_{x}^{1}(y-1)^{2}(y-2 x y-x) \phi(y) d y \equiv p_{1}(x)+q_{1}(x) .
\end{aligned}
$$

It is easy to verify that

$$
p_{1}(x)<0, \quad \forall 0<x \leq \frac{2}{3} ; \quad q_{1}(x)<0, \quad \forall \frac{1}{3} \leq x<1 .
$$

Hence $\phi^{\prime \prime}(x)<0$ for $1 / 3 \leq x \leq 2 / 3$, that is, $\phi^{\prime}(x)$ is strictly decreasing on $[1 / 3,2 / 3]$. By the above discussion we know that there exists $c \in(1 / 3,2 / 3)$ such that

$$
\phi^{\prime}(x)>0, \quad \forall 0<x<c ; \quad \phi^{\prime}(x)<0, \quad \forall c<x<1 .
$$

This completes the proof.

LEMmA 2.8 (see [5]). Let $P$ be a solid cone in $E, A: P \rightarrow P$ a completely continuous increasing operator. Suppose that

(i) there exist $u_{0}, v_{0} \in P$ such that $u_{0} \leq A u_{0}, A v_{0} \leq v_{0}, u_{0} \nless v_{0}$,

(ii) there exist $u^{*} \in P$ and $\delta>0$ such that $A u \geq \delta\|A u\| u^{*}$ for all $u \in P$,

(iii) $v_{0} \in \operatorname{Int} P$, and there exists $d>0$ such that $u^{*} \geq d u_{0}$.

Then A has a nonzero fixed point in $P$.

3. Main results. We first give a negative answer to Korman's conjecture by constructing a counterexample.

COUNTEREXAMPLE. Let $f(x, u)=u^{2}$ for all $x \in[0,1], 0 \leq u<+\infty$. If $\alpha \geq 2 \lambda_{1}$, $\gamma \geq 2 \lambda_{1}$, where $\lambda_{1}$ is given by (2.23), then (1.1) has no positive solutions.

Proof. By $\alpha \geq 2 \lambda_{1}, \gamma \geq 2 \lambda_{1}$ and (2.2) we have

$$
\xi(x) \geq \alpha(1-x)^{2}+\gamma x^{2} \geq 2 \lambda_{1}\left(1-2 x+2 x^{2}\right) \geq \lambda_{1}, \quad \forall x \in[0,1] .
$$

If $u$ is a positive solution of (1.1), that is, $u=A u$, then

$$
u(x)=A u(x)=\xi(x)+B u(x) \geq \xi(x) \geq \lambda_{1}, \quad \forall x \in[0,1] .
$$

So by (2.23) and Remark 2.2, we have

$$
\begin{aligned}
\int_{0}^{1} \phi(x) u(x) d x & =\int_{0}^{1} \phi(x) \xi(x) d x+\int_{0}^{1} \phi(x) d x \int_{0}^{1} G(x, y) u^{2}(y) d y \\
& =\int_{0}^{1} \phi(x) \xi(x) d x+\int_{0}^{1} u^{2}(y) d y \int_{0}^{1} G(y, x) \phi(x) d x \\
& \geq \int_{0}^{1} \phi(x) \xi(x) d x+\int_{0}^{1} \lambda_{1} u(y) d y \int_{0}^{1} G(y, x) \phi(x) d x \\
& \geq \lambda_{1} \int_{0}^{1} \phi(x) d x+\int_{0}^{1} \phi(y) u(y) d y .
\end{aligned}
$$


This is a contradiction. Hence $u \neq A u$ for all $u \in P$, that is, (1.1) has no positive solutions. This completes the proof.

Clearly, $f(x, u)=u^{2}$ satisfies all the conditions of the conjecture. So counterexample shows that Korman's conjecture is false. In what follows, we will investigate the existence of positive solutions of (1.1) by modifying the conditions of the conjecture. The following conditions will play roles in this section

$$
\begin{gathered}
\liminf \min _{x \in[0,1]} \frac{f(x, u)}{u}>\lambda_{1}, \quad \limsup _{u \rightarrow 0^{+}} \max _{x \in[0,1]} \frac{f(x, u+\xi(x))}{u}<\lambda_{1}, \\
\quad \limsup _{u \rightarrow+\infty} \max _{x \in[0,1]} \frac{f(x, u)}{u}<\lambda_{1}, \quad \liminf _{u \rightarrow 0^{+}} \min _{x \in[0,1]} \frac{f(x, u)}{u}>\lambda_{1},
\end{gathered}
$$

where $\lambda_{1}$ is given by (2.23).

$$
\sup _{u>0} \min _{x \in[0,1]} \frac{f(x, u)}{u}=+\infty, \quad \inf _{u \geq 2\|\xi\|} \max _{x \in[0,1]} \frac{f(x, u)}{u}=0 .
$$

THEOREM 3.1. Let (3.4) be satisfied. If $\xi(x) \equiv 0(x \in[0,1])$, then BVP (1.1) has at least one positive solution; if $\xi(x) \not \equiv 0(x \in[0,1])$, then $B V P(1.1)$ has at least two positive solutions.

Proof. Suppose that $v^{*}(x)$ is defined by (2.13), $\phi(x)$ and $\lambda_{1}$ are given by (2.23), and $\eta_{1} \equiv \int_{0}^{1} v^{*}(x) \phi(x) d x>0$. Denote

$$
Q=\left\{u \in P \mid \int_{0}^{1} u(x) \phi(x) d x \geq \eta\|u\|\right\}
$$

where $\eta=\min \left\{\eta_{1}, \int_{0}^{1} \phi(x) d x\right\}>0$. It is easy to see that $Q \neq \varnothing, Q \neq\{\theta\}$ is also a cone in $C[0,1]$.

We now show that for the operator $A$ defined by (2.1),

$$
A(P) \subset Q
$$

In fact, by Lemma 2.4,

$$
\int_{0}^{1} \xi(x) \phi(x) d x \geq \int_{0}^{1} v^{*}(x) \phi(x) d x \cdot \xi(y) \geq \eta \cdot \xi(y), \quad \forall y \in[0,1],
$$

that is, $\xi \in Q$.

By (2.23), Remark 2.2, (2.5), and Remark 2.5,

$$
\begin{aligned}
\phi(x) & =\lambda_{1} \int_{0}^{1} G(x, y) \phi(y) d y=\lambda_{1} \int_{0}^{1} G(y, x) \phi(y) d y \\
& \geq \lambda_{1} \int_{0}^{1} v^{*}(y) G(z, x) \phi(y) d y=\lambda_{1} \eta_{1} G(z, x), \quad \forall z, x \in[0,1] .
\end{aligned}
$$


Hence for $u \in P$,

$$
\begin{aligned}
\int_{0}^{1} \phi & (x) B u(x) d x \\
& =\int_{0}^{1} \phi(x) d x \int_{0}^{1} G(x, y) f(y, u(y)) d y=\int_{0}^{1} f(y, u(y)) d y \int_{0}^{1} G(y, x) \phi(x) d x \\
& =\frac{1}{\lambda_{1}} \int_{0}^{1} \phi(y) f(y, u(y)) d y \geq \frac{\lambda_{1} \eta_{1}}{\lambda_{1}} \int_{0}^{1} G(z, y) f(y, u(y)) d y \\
& \geq \eta B u(z), \quad \forall z \in[0,1],
\end{aligned}
$$

that is, $B u \in Q$. Therefore $A u=\xi+B u \in Q$.

By (3.4), there exist $R_{1}>\|\xi\|$ and $\varepsilon>0$ such that

$$
f(x, u) \geq\left(\lambda_{1}+\varepsilon\right) u, \quad \forall x \in[0,1], u \geq R_{1} .
$$

Hence, there exists a constant $C=C\left(R_{1}\right)>0$ such that

$$
f(x, u) \geq\left(\lambda_{1}+\varepsilon\right) u-C, \quad \forall x \in[0,1], u \geq 0 .
$$

Choose $u_{0} \in Q \backslash\{\theta\}$, we assert that for any fixed $R>\max \left\{R_{1},\|\xi\|+1, C /(\eta \varepsilon)\right\}>0$,

$$
u-A u \neq \lambda u_{0}, \quad \forall u \in P,\|u\|=R, \lambda \geq 0 .
$$

Otherwise, suppose that there exist $u_{1} \in P,\left\|u_{1}\right\|=R$ and $\lambda_{0} \geq 0$ such that $u_{1}-A u_{1}=$ $\lambda_{0} u_{0}$, then by (3.13), Remark 2.2, and (2.23) we have

$$
\begin{aligned}
0 & \geq-\lambda_{0} \int_{0}^{1} u_{0}(x) \phi(x) d x=\int_{0}^{1} \phi(x) A u_{1}(x) d x-\int_{0}^{1} \phi(x) u_{1}(x) d x \\
& \geq \int_{0}^{1} \phi(x) B u_{1}(x) d x-\int_{0}^{1} \phi(x) u_{1}(x) d x \\
& \geq \int_{0}^{1} \phi(x) d x \int_{0}^{1} G(x, y)\left[\left(\lambda_{1}+\varepsilon\right) u_{1}(y)-C\right] d y-\int_{0}^{1} \phi(x) u_{1}(x) d x \\
& =\int_{0}^{1}\left[\left(\lambda_{1}+\varepsilon\right) u_{1}(y)-C\right] d y \int_{0}^{1} G(y, x) \phi(x) d x-\int_{0}^{1} \phi(x) u_{1}(x) d x \\
& =\frac{\lambda_{1}+\varepsilon}{\lambda_{1}} \int_{0}^{1} u_{1}(y) \phi(y) d y-\frac{C}{\lambda_{1}} \int_{0}^{1} \phi(y) d y-\int_{0}^{1} \phi(x) u_{1}(x) d x \\
& =\frac{\varepsilon}{\lambda_{1}} \int_{0}^{1} u_{1}(y) \phi(y) d y-\frac{C}{\lambda_{1}} \int_{0}^{1} \phi(y) d y .
\end{aligned}
$$

Observing $u_{1}=A u_{1}+\lambda_{0} u_{0} \in Q$, we have

$$
\frac{C}{\varepsilon} \geq \frac{C}{\varepsilon} \int_{0}^{1} \phi(y) d y \geq \int_{0}^{1} u_{1}(y) \phi(y) d y \geq \eta\left\|u_{1}\right\|=\eta R,
$$

in contradiction with $R>C /(\varepsilon \eta)$. Hence (3.14) holds. 
Denote

$$
P_{\xi}=\{u(x)+\xi(x) \mid u \in P\}, \quad \Omega_{R}=P_{\xi} \cap U_{R} .
$$

Then $A: P \rightarrow P_{\xi}$ is completely continuous. Without loss of generality, suppose that $A u \neq u$ for $u \in \partial U_{R} \cap P_{\xi}$. Therefore the fixed point index $i\left(A, \Omega_{R}, P_{\xi}\right)$ is well defined (see $[1,2])$. We now prove that

$$
i\left(A, \Omega_{R}, P_{\xi}\right)=0 .
$$

Let $u_{0}$ be given as above, denote $a=\left\|u_{0}\right\|, b=\sup _{\bar{\Omega}_{R}}\|A u\|$. Choose $\rho>0$ sufficiently large such that $\rho a>b+R$. Then it follows from (3.14) and the homotopy invariance of the fixed point index that

$$
i\left(A, \Omega_{R}, P_{\xi}\right)=i\left(A+\rho u_{0}, \Omega_{R}, P_{\xi}\right) .
$$

The latter equals 0 since for any $u \in \bar{\Omega}_{R}$

$$
\left\|A u+\rho u_{0}\right\| \geq \rho\left\|u_{0}\right\|-\|A u\| \geq \rho a-b>R \geq\|u\| .
$$

Thus (3.18) holds.

On the other hand, by (3.4), there exist $0<r<\min \left\{R_{1}-\|\xi\|, 1\right\}$ and $\varepsilon_{1}>0$ sufficiently small such that

$$
f(x, u+\xi(x)) \leq\left(\lambda_{1}-\varepsilon_{1}\right) u, \quad \forall x \in[0,1], 0 \leq u \leq r .
$$

We now prove that

$$
\xi+t B u \neq u, \quad \forall t \in[0,1], u \in P_{\xi},\|u-\xi\|=r .
$$

In fact, if $t_{1} \in[0,1], u_{2} \in P \xi,\left\|u_{2}-\xi\right\|=r$ satisfy $\xi+t_{1} B u_{2}=u_{2}$, then by (2.23), and (3.21) we have

$$
\begin{aligned}
\int_{0}^{1} \phi & (x) u_{2}(x) d x \\
& =t_{1} \int_{0}^{1} \phi(x) d x \int_{0}^{1} G(x, y) f\left(y, u_{2}(y)\right) d y+\int_{0}^{1} \phi(x) \xi(x) d x \\
& \leq \int_{0}^{1} \phi(x) d x \int_{0}^{1} G(x, y) f\left(y,\left(u_{2}(y)-\xi(y)\right)+\xi(y)\right) d y+\int_{0}^{1} \phi(x) \xi(x) d x \\
& \leq\left(\lambda_{1}-\varepsilon_{1}\right) \int_{0}^{1}\left(u_{2}(y)-\xi(y)\right) d y \int_{0}^{1} G(x, y) \phi(x) d x+\int_{0}^{1} \phi(x) \xi(x) d x \\
& =\frac{\lambda_{1}-\varepsilon_{1}}{\lambda_{1}} \int_{0}^{1}\left(u_{2}(y)-\xi(y)\right) \phi(y) d y+\int_{0}^{1} \phi(x) \xi(x) d x<\int_{0}^{1} \phi(y) u_{2}(y) d y,
\end{aligned}
$$

a contradiction. Therefore (3.22) holds. By the homotopy invariance of the fixed point index, we have

$$
i\left(A, \Omega_{r}, P_{\xi}\right)=i\left(\xi, \Omega_{r}, P_{\xi}\right)=1,
$$

where $\Omega_{r}=P_{\xi} \cap\{u \mid\|u-\xi\|<r\}$. Obviously, $\bar{\Omega}_{r} \subset \Omega_{R}$. 
By (3.18), (3.24), and the additivity of the fixed point index we get

$$
i\left(A, \Omega_{R} \backslash \bar{\Omega}_{r}, P_{\xi}\right)=-1,
$$

which means that $A$ has a fixed point in $\Omega_{R} \backslash \bar{\Omega}_{r}$. Also, by (3.24), $A$ has a fixed point in $\Omega_{r}$.

Consequently, if $\xi(x) \not \equiv 0(x \in[0,1])$, that is, $\theta \notin P_{\xi}$, then $A$ has at least two fixed points in $P_{\xi}$, that is, (1.1) has at least two positive solutions; if $\xi(x) \equiv 0(x \in[0,1])$, then $A$ has at least one nonzero fixed point in $P_{\xi}$, that is, (1.1) has at least one positive solution. This completes the proof.

In a similar way as proving Theorem 3.1, we obtain the following theorem.

THEOREM 3.2. If (3.5) is satisfied, then BVP (1.1) has at least one positive solution.

REMARK 3.3. Theorems 3.1 and 3.2 cover the superlinear and the sublinear nonlinearities, respectively. As is well known that conditions (3.4) and (3.5) expressed by $\lambda_{1}$ are more general than those expressed by 0 and $+\infty$, to say, Theorem 3.2 improves [3, Theorem 3]. Furthermore, it is clear that Theorem 3.1 remains valid if we replace the second inequality in (3.4) by

$$
\limsup _{u \rightarrow 0^{+}} \max _{0 \leq x \leq 1,0 \leq v \leq\|\xi\|} \frac{f(x, u+v)}{u}<\lambda_{1}
$$

It is easy to give some simple functions $f$, which satisfy (3.4) and the above inequality. For example

$$
f(x, u)= \begin{cases}0, & 0 \leq u \leq\|\xi\|, \\ (u-\|\xi\|)^{2}, & u>\|\xi\| .\end{cases}
$$

THEOREM 3.4. Let $f(x, u)$ be increasing in $u$. If (3.6) is satisfied, then BVP (1.1) has at least one positive solution.

Proof. Let $P$ be the cone of $C[0,1]$ defined in Section 2, then $P$ is normal and solid (see [1, 2]). Let $A$ and $B$ be defined by (2.1) and (2.3), respectively, then $A, B: P \rightarrow P$ are completely continuous operators. Also, $A$ and $B$ are increasing since $f(x, u)$ is increasing in $u$.

By condition (3.6), there exists $\bar{u}>0$ such that

$$
f(x, \bar{u})>\frac{\lambda_{1}}{\phi_{0} \tau_{0} g_{0}} \bar{u}, \quad \forall x \in[0,1]
$$

where $\phi_{0}$ is given by (2.24), $\tau_{0}$ is given by (2.15) and $g_{0}=\int_{1 / 3}^{2 / 3} G(1 / 2, y) d y>0$. We assert that

$$
\frac{\bar{u}}{\phi_{0}} \phi(x) \leq B\left(\frac{\bar{u}}{\phi_{0}} \phi(x)\right)
$$


where $\phi$ is given by (2.23). In fact, by (2.5), (2.15), (2.23), (2.24), and (3.28) we have

$$
\begin{aligned}
B\left(\frac{\bar{u}}{\phi_{0}} \phi(x)\right) & =\int_{0}^{1} G(x, y) f\left(y, \frac{\bar{u}}{\phi_{0}} \phi(y)\right) d y \geq \int_{1 / 3}^{2 / 3} G(x, y) f\left(y, \frac{\bar{u}}{\phi_{0}} \phi(y)\right) d y \\
& \geq \int_{1 / 3}^{2 / 3} G(x, y) f(y, \bar{u}) d y \geq \frac{\lambda_{1}}{\phi_{0} \tau_{0} g_{0}} \int_{1 / 3}^{2 / 3} G(x, y) d y \cdot \bar{u} \\
& \geq \frac{\lambda_{1} \bar{u}}{\phi_{0} \tau_{0} g_{0}} \int_{1 / 3}^{2 / 3} v^{*}(x) G(z, y) d y \geq \frac{\lambda_{1} \bar{u}}{\phi_{0} g_{0}} \int_{1 / 3}^{2 / 3} G(z, y) d y \int_{0}^{1} G(x, t) d t \\
& \geq \frac{\lambda_{1} \bar{u}}{\phi_{0} g_{0}} \int_{1 / 3}^{2 / 3} G(z, y) d y \int_{0}^{1} G(x, t) \phi(t) d t=\frac{\bar{u}}{\phi_{0} g_{0}} \int_{1 / 3}^{2 / 3} G(z, y) d y \cdot \phi(x) \\
& =\frac{1}{g_{0}} \int_{1 / 3}^{2 / 3} G(z, y) d y \cdot \frac{\bar{u}}{\phi_{0}} \phi(x), \quad \forall x, z \in[0,1] .
\end{aligned}
$$

Hence (3.29) holds. Denote $u_{0}(x)=B\left(\left(\bar{u} / \phi_{0}\right) \phi(x)\right)$, then

$$
A u_{0}(x)=\xi(x)+B u_{0}(x) \geq B\left(B\left(\frac{\bar{u}}{\phi_{0}} \phi(x)\right)\right) \geq B\left(\frac{\bar{u}}{\phi_{0}} \phi(x)\right)=u_{0}(x),
$$

that is, $u_{0}(x)$ is a subsolution of $A$.

On the other hand, by (3.6), there exists a constant $\bar{v}>2\|\xi\|$ such that

$$
f(x, \bar{v})<\frac{\bar{v}}{2 g_{1}}, \quad \forall x \in[0,1],
$$

where $g_{1}=\max _{x \in[0,1]} \int_{0}^{1} G(x, y) d y$. Then

$$
A \bar{v}(x)=\xi(x)+B \bar{v}(x) \leq \xi(x)+\int_{0}^{1} G(x, y) d y \cdot \frac{\bar{v}}{2 g_{1}} \leq \xi(x)+\frac{\bar{v}}{2} \leq \bar{v}
$$

that is, $\bar{v}$ is a supersolution of $A$.

(i) If $u_{0} \leq \bar{v}$, then it follows from (3.31), (3.33), and [1, Theorem 19.1] that $A$ has a fixed point $w^{*}(x)$ satisfying $u_{0} \leq w^{*} \leq \bar{v}$.

(ii) If $u_{0} \npreceq \bar{v}$, then define

$$
P\left(v^{*}\right)=\left\{u \in P \mid u(x) \geq\|u\| v^{*}(x), \forall x \in[0,1]\right\},
$$

where $v^{*}$ is given by (2.13). We show that $A$ maps $P$ into $P\left(v^{*}\right)$. In fact, for any $u \in P$, we have by (2.5)

$$
\begin{aligned}
B u(x) & =\int_{0}^{1} G(x, y) f(y, u(y)) d y \\
& \geq \int_{0}^{1} v^{*}(x) G(z, y) f(y, u(y)) d y=v^{*}(x) B u(z), \quad \forall x, z \in[0,1] .
\end{aligned}
$$


Hence by Lemma 2.4 and Remark 2.5, we have

$$
A u(x)=\xi(x)+B u(x) \geq v^{*}(x) \xi(y)+v^{*}(x) B u(y)=v^{*}(x) A u(y), \quad \forall x, y \in[0,1] .
$$

Thus $A u(x) \geq\|A u\| v^{*}(x)(\forall x \in[0,1])$, that is, Lemma 2.8(ii) is satisfied for $u^{*}(x)$ $=v^{*}(x), \delta=1$. Moreover, $\bar{v} \in \operatorname{Int} P$, and by (2.14), $v^{*}(x) \geq \tau B\left(\left(\bar{u} / \phi_{0}\right) \phi(x)\right)=$ $\tau u_{0}(x)$ for some $\tau>0$, that is, Lemma 2.8(iii) holds. Therefore, by (3.31), (3.33), and Lemma 2.8, $A$ has a nonzero fixed point in $P$. This completes the proof.

REMARK 3.5. In a similar way as establishing Lemmas 2.3, 2.6, 2.7 and Theorems 3.1, 3.2 and 3.4, one can also discuss the existence of positive solutions for other physically important boundary conditions for $L u=u^{\prime \prime \prime \prime}$.

ACKNOWLEDGEMENT. The author is supported in part by Monbusho Scholarship of Japan, National Natural Science Foundation of China and the State Education Commission Doctoral Foundation of China.

\section{REFERENCES}

[1] K. Deimling, Nonlinear Functional Analysis, Springer-Verlag, Berlin, 1985. MR 86j:47001. Zbl 559.47040.

[2] D. J. Guo and V. Lakshmikantham, Nonlinear Problems in Abstract Cones, Notes and Reports in Mathematics in Science and Engineering, vol. 5, Academic Press, Massachusetts, 1988. MR 89k:47084. Zbl 661.47045.

[3] P. Korman, Computation of displacements for nonlinear elastic beam models using monotone iterations, Int. J. Math. Math. Sci. 11 (1988), no. 1, 121-128. MR 88j:65282. Zbl 631.73073 .

[4] R. D. Nussbaum, Eigenvectors of nonlinear positive operators and the linear Kreĭn-Rutman theorem, Fixed Point Theory (Sherbrooke, Que., 1980), Lecture Notes in Math., vol. 886, Springer, Berlin, 1981, pp. 309-330. MR 83b:47068. Zbl 489.47037.

[5] J. X. Sun, Some new fixed point theorems of increasing operators and applications, Appl. Anal. 42 (1991), no. 3-4, 263-274. MR 92g:47087. Zbl 738.47052.

Bendong Lou: Department of Mathematics, Shandong University, JinAn 250100, CHINA

Current address: Graduate School of Mathematical Sciences, The University of TOKYO, KOMABA 3-8-1, MEgURO-KU, TOKYO 153-8914, JAPAN

E-mail address: blou@glacia.ms.u-tokyo.ac.jp 


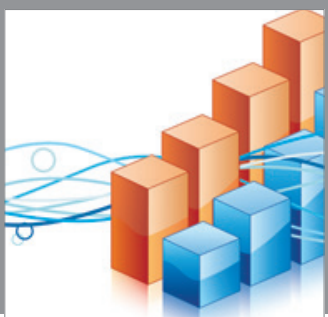

Advances in

Operations Research

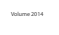

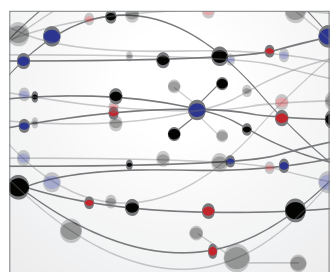

\section{The Scientific} World Journal
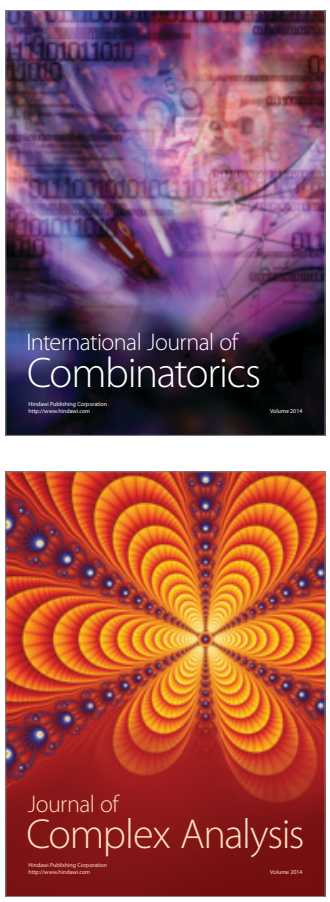

International Journal of

Mathematics and

Mathematical

Sciences
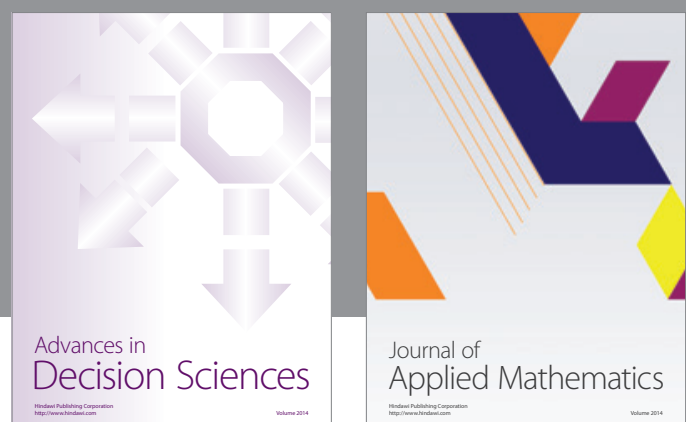

Journal of

Applied Mathematics
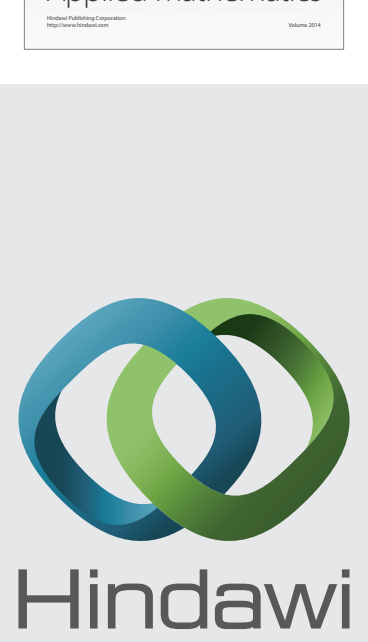

Submit your manuscripts at http://www.hindawi.com
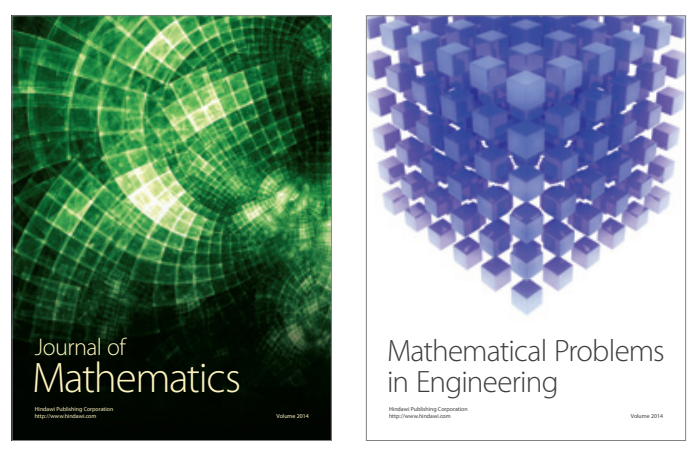

Mathematical Problems in Engineering
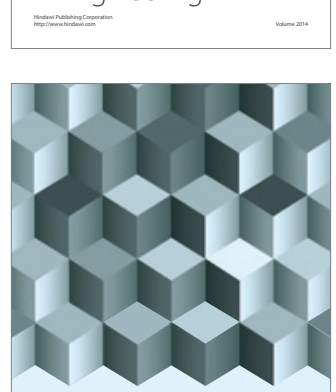

Journal of

Function Spaces
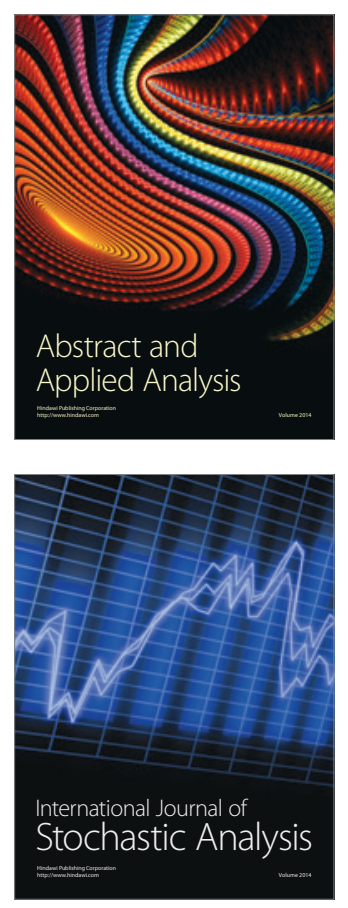

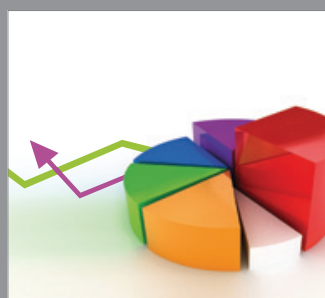

ournal of

Probability and Statistics

Promensencen
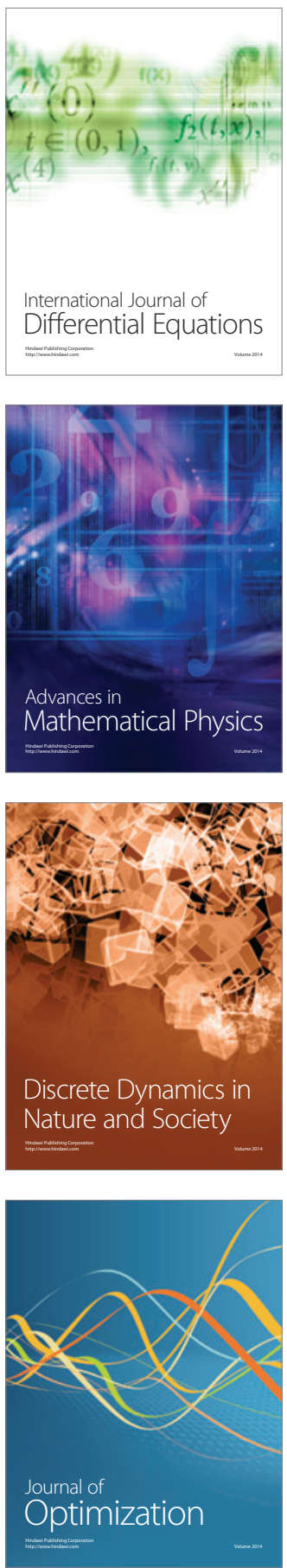